Short communication

\title{
Absorbance enhancement in microplate wells for improved-sensitivity biosensors
}

\author{
Guillaume Suárez $^{\text {a,* }}$, Christian Santschi ${ }^{\mathrm{b}}$, Gregory Plateel ${ }^{\mathrm{a}}$, \\ Olivier J.F. Martin ${ }^{\mathrm{b}}$, Michael Riediker ${ }^{\mathrm{a}}$ \\ a Institute for Work and Health, Route de la Corniche 2, CH-1066 Epalinges-Lausanne, Switzerland \\ ${ }^{\mathrm{b}}$ Nanophotonics and Metrology Laboratory, Swiss Federal Institute of Technology Lausanne (EPFL), EPFL-STI-NAM, \\ Station 11, CH-1015 Lausanne, Switzerland
}

\section{A R T I C L E I N F O}

\section{Article history:}

Received 8 October 2013

Received in revised form

9 December 2013

Accepted 26 December 2013

Available online 18 January 2014

Keywords:

Absorbance enhancement

Optical biosensor

Hydrogen peroxide

Random medium

Multiwell microplate

\begin{abstract}
A B S T R A C T
A generic optical biosensing strategy was developed that relies on the absorbance enhancement phenomenon occurring in a multiple scattering matrix. Experimentally, inserts made of glass fiber membrane were placed into microplate wells in order to significantly lengthen the trajectory of the incident light through the sample and therefore increase the corresponding absorbance. Enhancement factor was calculated by comparing the absorbance values measured for a given amount of dye with and without the absorbanceenhancing inserts in the wells. Moreover, the dilution of dye in solutions with different refractive indices $(R I)$ clearly revealed that the enhancement factor increased with the $\Delta R I$ between the membrane and the surrounding medium, reaching a maximum value $(E F>25)$ when the membranes were dried. On this basis, two $\mathrm{H}_{2} \mathrm{O}_{2}$-biosensing systems were developed based on the biofunctionalization of the glass fiber inserts either with cytochrome $c$ or horseradish peroxidase (HRP) and the analytical performances were systematically compared with the corresponding bioassay in solution. The efficiency of the absorbance-enhancement approach was particularly clear in the case of the cytochrome $c$-based biosensor with a sensitivity gain of 40 folds and wider dynamic range. Therefore, the developed strategy represents a promising way to convert standard colorimetric bioassays into optical biosensors with improved sensitivity.
\end{abstract}

(c) 2014 Elsevier B.V. All rights reserved

\section{Introduction}

Colorimetric detection technologies provide to the analyst a valuable set of tools suitable in performing routine tests for biomedical diagnosis or environmental monitoring purposes. Despite their diversity in terms of format - ranging from standard cuvettes (Kuang et al., 2007) to multiwell microplates (Gillespie and Ainsworth, 2007), optical fibers (Malcik et al., 2005) or dipsticks (Liu et al., 2006) - the detection principle indistinctively bares on measuring changes in absorbance, as the physical response to a specific chemical reaction or biological recognition event. The most popular representative of colorimetric tests is the so-called enzymelinked immunosorbent assay, referred to as ELISA, which combines high throughput and high sensitivity for quantitative or semiquantitative detection of a large number of analytes (Martinez, 2011; Plested et al., 2003). Here, the measured absorbance change relies on the enzymatic conversion of a substrate into a chromogenic compound. Recently, the integration of novel materials in colorimetric detection systems has enlarged their capabilities thanks to their conformation-dependent absorption properties or pseudo-enzymatic catalytic behavior (Song et al., 2011). Typically, for a given system

\footnotetext{
* Corresponding author. Tel.: +4121314 58 97; fax: +41213147421.

E-mail address: guillaume.suarez@chuv.ch (G. Suárez).
}

the absorbance change consecutive to the assembly (or disassembly) of functionalized gold nanoparticles (NPs) in a colloidal solution provides a sensitive indication on the presence of chemical (e.g. metal ions (Liu and Lu, 2003) or pH (Chen et al., 2008)) or biological (e.g. ssDNA (Mao et al., 2009; Sato et al., 2003), adenosine (Liu et al., 2006)) factors that control this mechanism. On the other side, some metal oxide NPs such as iron oxide $\left(\mathrm{Fe}_{3} \mathrm{O}_{4}\right)$ have been shown to exhibit an intrinsic mimetic peroxidase activity (Gao et al., 2007) which enabled their use as detection labels in ELISA or in $\mathrm{H}_{2} \mathrm{O}_{2}$-based melamine sensors (Ding et al., 2010). In order to improve the analytical performances of colorimetric detection systems the main effort has been focused at the reaction/recognition level by seeking catalysts with higher reaction rates and recognition biomolecules with higher affinity constants. However, another track for sensitivityenhancement strategies is clearly indicated by the physical nature of absorbance, governed by the Beer-Lambert law

$A=\varepsilon l C$

where being the molar absorptivity is $\varepsilon$, inherent to the species which is found at the molar concentration $C$ in the sample, while the path length of light, $l$, remains an adaptable parameter.

Therefore, an interesting perspective to develop colorimetric assays with improved sensitivity resides in the ability to lengthen the trajectory of light through the sample. Such an approach is manifested in the detection systems based on either long path 
flow cell where the light is guided through a few meters long capillary filled with the assay mixture (Zhang, 2006) or on-chip lateral beam guidance device particularly suitable for low-aspectratio sensing chambers (Grumann et al., 2006). However, in these strategies the gain in sensitivity is due to the fact that a larger volume of the sample - that is a higher number of absorbing molecules - is getting probed by the light. In other words, in such approaches the intrinsic sensitivity understood as the amplitude of signal generated per mole of absorber remains theoretically unchanged. In contrast, we have recently reported that for the absorption spectrum of a given biomolecule loaded into a random medium, such as cytochrome $c$ embedded into an aggregate of polystyrene beads, absorbance values are enhanced due to the elongation of the light optical path (Suárez et al., 2012, 2013). In those preliminary studies the cytochrome $c$ absorbance enhancement was efficiently used to develop a sensitive photonic biosensor able to detect in real-time the release of $\mathrm{H}_{2} \mathrm{O}_{2}$ by cells under oxidative stress. In the present work, we further extend the biodetection approach to the use of protein-functionalized glass fiber membranes as absorbance enhancing inserts adapted to microplate wells and which exhibit improved sensitivity compared to their corresponding bioassays in solution. In addition, this study experimentally proves the dependence of such an absorbance enhancement on the refractive index difference, $\Delta R I$, between the scattering matrix and the surrounding medium.

\section{Materials and methods}

\subsection{Chemicals}

Horseradish peroxidase (HRP type VI, 250-320 $\mathrm{U} \mathrm{mg}_{\text {solid }}{ }^{-1}$ ), cytochrome $c$ from bovine heart ( $\geq 95 \%$ ), glutaraldehyde solution $25 \%$, hydrogen peroxide (30\%), 3-amino-9-ethylcarbazole (AEC), bromocresol green sodium salt (BCG), ethanol, sodium ascorbate, phosphate disodic, phosphate monosodic, sodium chloride, calcium chloride and sodium acetate were all purchased from SigmaAldrich (Buchs, Switzerland).

\subsection{Materials}

Clear polystyrene flat bottom 96-well microplates were provided by Corning (Corning Inc., NY, USA). For the insert-based biosensor approach a circular aperture was drilled at the bottom of the wells bottom using a $2 \mathrm{~mm}$ diameter drill bit. Absorbanceenhancer inserts were cut out from glass fiber membranes GF/B (1 mm thickness) purchased from Membrane Solutions (North Bend, USA).

\subsection{Glass fiber membrane loaded with $B C G$}

In the experiments involving BCG, clean membrane inserts are placed in standard microplate wells before loading with $25 \mu \mathrm{L}$ of BCG solution $(120 \mu \mathrm{M})$ containing different amounts of glucose from $0 \%$ to $80 \%$.

\subsection{Biofunctionalization glass fiber membranes}

Enhancer inserts made from glass fiber membrane are placed in the wells of the modified microplate (with bottom aperture) and are subsequently biofunctionalized with either cytochrome $c$ or HRP via the following protocol: $25 \mu \mathrm{L}$ of the protein solution ( $1 \mathrm{mg} \mathrm{mL}^{-1}$ HRP or $25 \mu \mathrm{M}$ of cytochrome c) containing $0.5 \%$ of glutaraldehyde is added immediately after preparation onto each clean membrane insert and left to react for $30 \mathrm{~min}$ at room temperature. Then, the protein-modified membrane inserts are rinsed intensively by flowing several times PBS pH 7.2 or acetate buffer $\mathrm{pH}$ 5.0, for cytochrome $c$ and HRP, respectively. In the case of cytochrome $c$-based biosensor, the heme-group was getting reduced prior to measurements by flowing several times $100 \mu \mathrm{L}$ of ascorbic acid (1 $\mathrm{mM}$ in PBS) through the modified membrane. The cytochrome $c$-membranes are finally rinsed intensively with PBS solution.

\subsection{Absorbance measurements}

All the absorbance measurements are performed on a Infinite ${ }^{\circledR}$ 200 PRO multimode microplate reader from Tecan Group Ltd.

\section{a}

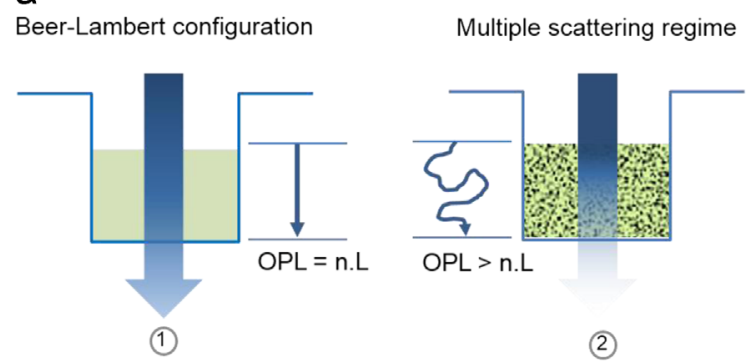

b

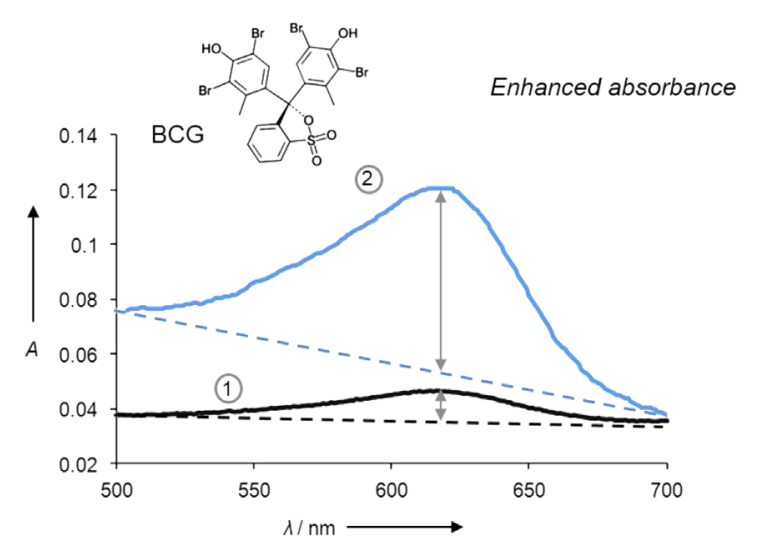

C

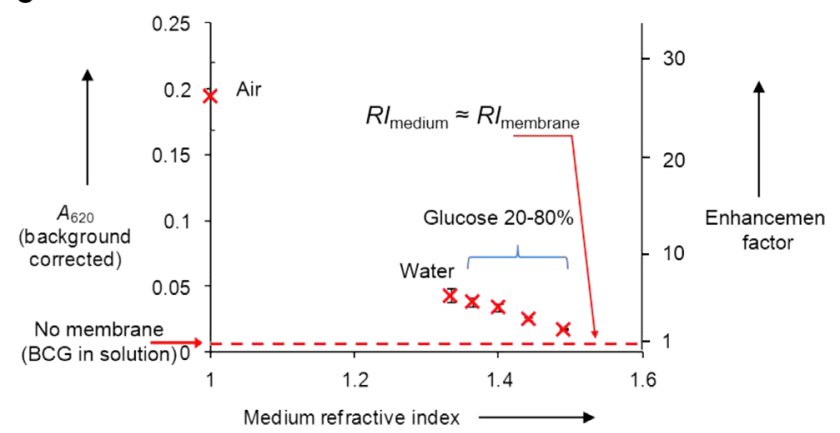

Fig. 1. (a) Detection principle based on the enhancement of the absorbance for a given molecule loaded in a random medium due to the elongation of the optical path length under multiple scattering regime. In comparison to the standard BeerLambert configuration, where the absorbance measurements are performed in solution (1), a significant enhancement is observed when the molecule is loaded into a strongly scattering medium (2). (b) Experimentally, the absorbance spectra of bromocresol green ( $3 \mathrm{nmol}$ BCG) were measured in aqueous solution (1) and loaded into a glass fiber membrane (2). The height of BCG absorption peak $(621 \mathrm{~nm})$ was calculated (baseline between 500 and $700 \mathrm{~nm}$ ) for varying values of $\Delta R I=R i_{\text {membrane }}-R I_{\text {medium. }}$ (c) The corresponding plot clearly indicates that the absorbance enhancement factor increases with $\Delta R I$ (maximum enhancement for membrane/air configuration). Ideally, there is no absorbance enhancement $(E F=1)$ when $R I_{\text {medium }}$ is close to the value corresponding to the membrane material (i.e. borosilicate, $R I=1.54$ ). 
The optimal reading wavelengths for BCG, HRP/AEC and cytochrome $c$ are 621, 580 and 542/550/556 nm, respectively.

\section{Results and discussion}

As depicted in Fig. 1 the signal amplification principle relies on physical properties of the light propagating into strongly scattering materials (Svensson and Shen, 2010). In the classic bioassay configuration light travels in a straight line through the absorbercontaining solution and the resulting absorbance, at a given wavelength, varies linearly with both the path length of the sample and the concentration of the absorber, as stated by the Beer-Lambert law (Eq. (1)). In contrast, the propagation of light into a non-uniform material is controlled by a multiple scattering regime and its path length then largely exceeds the geometrical dimension of the sample (John and Pang, 1996; Popescu and Dogariu, 1999). Consequently to the path length amplification due to light diffusion in strongly scattering medium, the absorbance measured for a given amount of absorber in the sample gets enhanced. Such absorbance enhancement effect is shown in Fig. 1b, where the optical spectrum obtained for $120 \mu \mathrm{M}$ (3 nmol) of bromocresol green (BCG) loaded into a glass fiber membrane insert ( $1 \mathrm{~mm}$ thickness) results in greater absorption than the one corresponding to the same amount of BCG in aqueous solution. The signal baseline registered for the membrane (no BCG) was also increased as a consequence of the multiscattering (light loss) and therefore in Fig. 1b the background was corrected to improve the readability of the graph. In order to further characterize the behavior of this absorbance enhancement another experiment was conducted in which BCG was prepared in aqueous solutions exhibiting different values of RI. The addition of increasing amount of sucrose in water $(0 \%, 20 \%, 40 \%, 60 \%$ and $80 \%)$ gave rise to BCG solutions with the $R I$ values of $1.333,1.364,1.400,1.442$ and 1.490 , respectively. Considering the $R I$ value of the glass fiber membrane (borosilicate), $R I_{\text {membrane }}=1.54$, it is interesting to notice that the absorbance peak value corresponding to BCG $(621 \mathrm{~nm})$ decreases almost linearly when $R I_{\text {medium }}$ gets closer to $R I_{\text {membrane. }}$ Furthermore, the extrapolation of the linear dependence to the point at which $R I_{\text {membrane }}=\mathrm{RI}_{\text {medium }}(\Delta R I=0)$ gives a peak height value close to the one obtained for BCG in water and in the absence of the membrane. At this particular point the membrane becomes transparent and the membrane/medium system can now be defined as a uniform medium where propagating light no longer gets scattered. The enhancement factor $(E F)$ is 1 at $\Delta R I=0$ and then progressively increases with $\Delta R I$ (i.e. when $R I$ medium becomes lower) until it reaches a maximum value of almost $E F=30$ when the membrane initially loaded with BCG in water is left to dry for $48 \mathrm{~h}\left(\Delta R I_{\max }\right)$. In the case of medium=air the absorbance response is higher than what should be expected from the extrapolation of the dependence obtained for water/sucrose system. This can be due to some changes taking place in the geometrical characteristics of the membrane during the drying process (e.g. pore size, thickness) which affect the modification of the light path length. However, for a given membrane geometry the observed dependence of the absorbance enhancement on the heterogeneity of the system in terms of $\Delta R I$ at the membrane/ medium interfaces clearly confirms that the elongation of the light path length generated under a multiple scattering regime is responsible for the observed enhancement phenomenon.

On the basis of this absorbance enhancement principle, and as a proof of concept, a versatile detection system was established which enabled the development of two $\mathrm{H}_{2} \mathrm{O}_{2}$ biosensors with improved sensitivity in a 96-well microplate format. As depicted in Fig. 2, enhancer inserts made from glass fiber membrane are biofunctionalized with the recognition biomolecule of interest here an $\mathrm{H}_{2} \mathrm{O}_{2}$-sensitive enzyme - in the presence of a chemical crosslinker ( $0.5 \%$ glutaraldehyde). The bio-active enhancer inserts

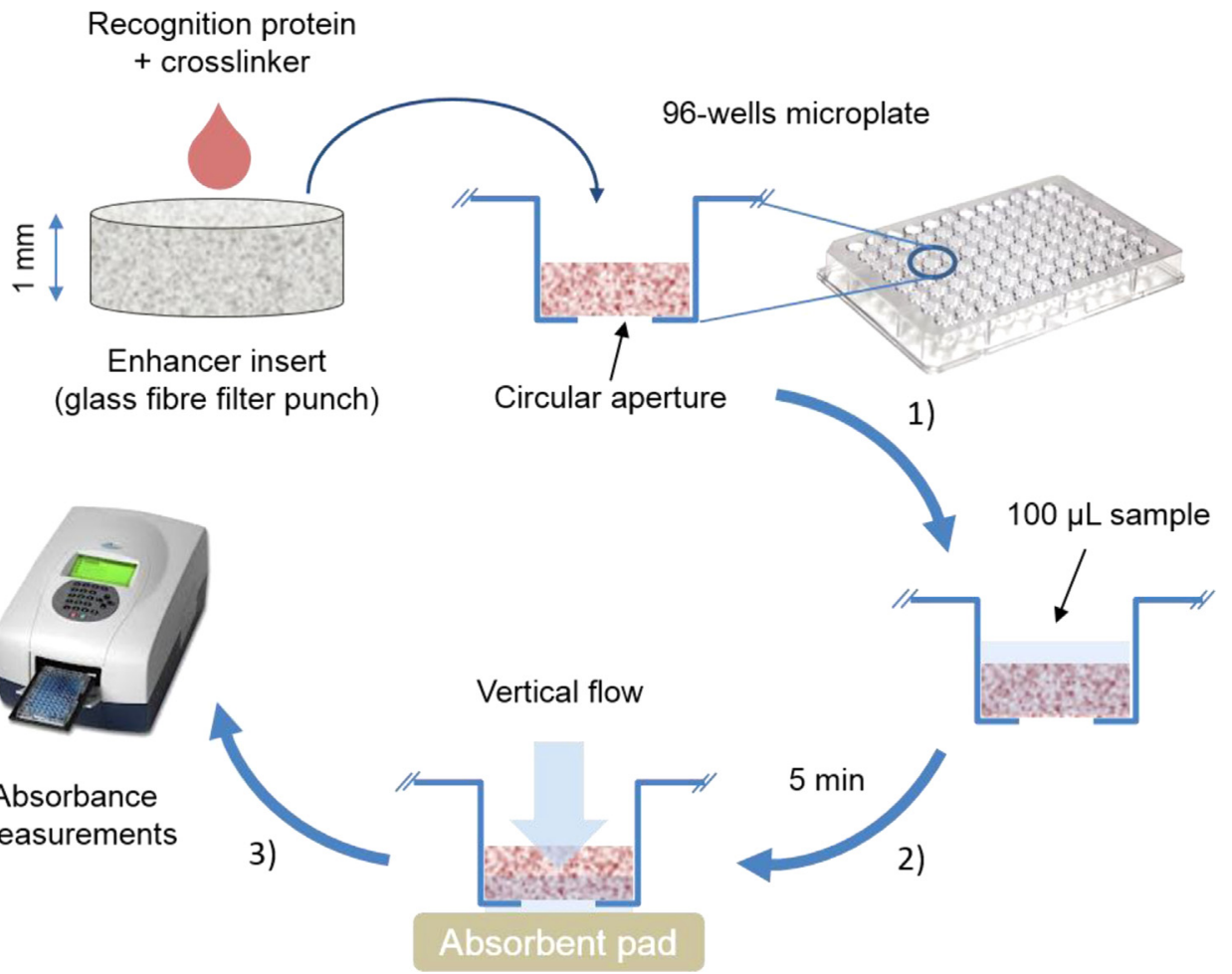

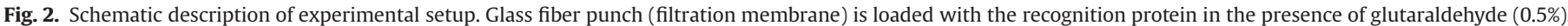

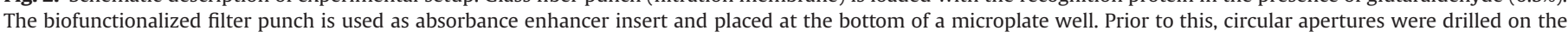

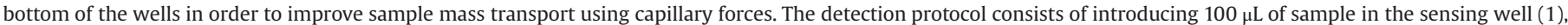
which is then let to flow through the insert after $5 \mathrm{~min}$ (2). Changes in absorbance are measured using a standard microplate reader spectrometer (3). 
are introduced into clean wells on the bottom of which circular apertures have priorly been drilled at the center $(1.5 \mathrm{~mm}$ diameter). The detection protocol consists of adding $100 \mu \mathrm{L}$ of sample mixture into each well modified with insert and holding for 5 min. Then, a vertical flow is generated by putting the microplate in contact with an absorbent pad (driven by capillary forces), forcing the whole sample volume to get through the analyte-sensitive insert. Finally, absorbance measurements are performed on each individual well using a standard microplate reader. Noticeably, the term biosensor is voluntarily used here as it refers to a detection system where the recognition biomolecule $\left(\mathrm{H}_{2} \mathrm{O}_{2}\right.$-sensitive protein) is immobilized on the transducer surface (membrane glass fibers), in contrast to bioassay in which all the (bio)reagents stand in solution (Renneberg et al., 2008).

The first biosensor configuration described in Fig. 3 relies on measuring the oxidation of cytochrome $c$, a globular hemoprotein (12.4 kDa), that occurs in the presence of $\mathrm{H}_{2} \mathrm{O}_{2}$. This ability of $\mathrm{H}_{2} \mathrm{O}_{2}$ to oxidize ferrocytochrome $c$ has been first shown by Vandewalle

\section{a}

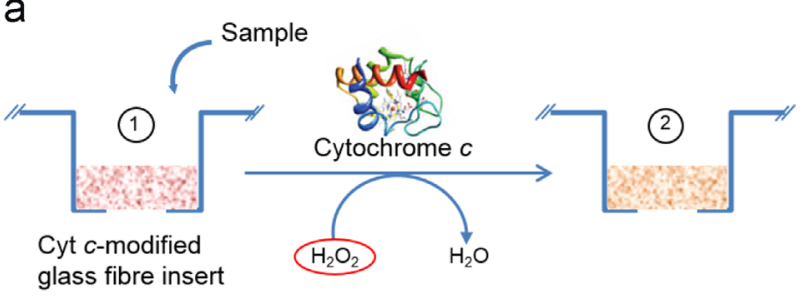

b

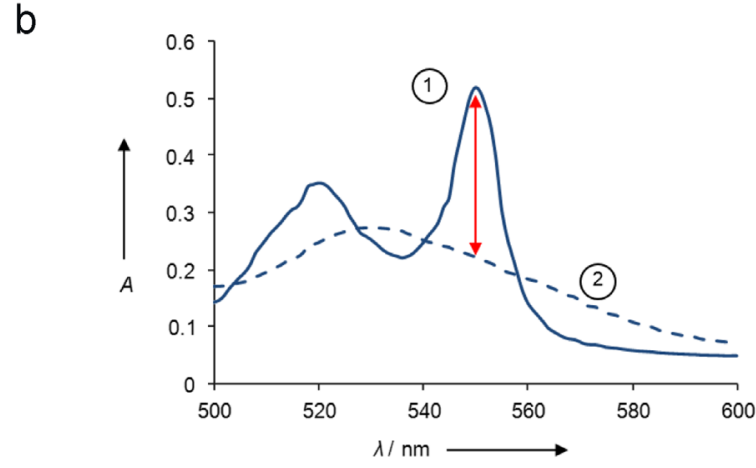

C

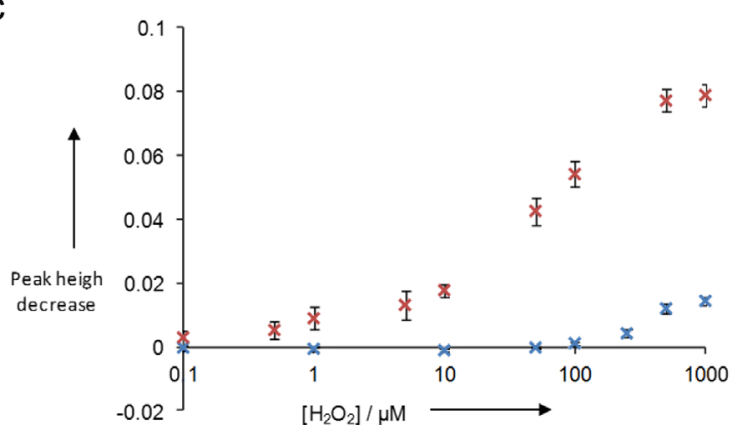

Fig. 3. (a) Biosensor configuration for $\mathrm{H}_{2} \mathrm{O}_{2}$ detection based on the enhanced absorbance measurement of cytochrome $c$ oxidation. Clean glass fiber inserts are modified with reduced cytochrome $c$ and washed several times prior to experiments. (b) Cytochrome $c$ oxidation in the presence of $\mathrm{H}_{2} \mathrm{O}_{2}$ induces a decrease in the absorption peak at $550 \mathrm{~nm}$ (baseline between 542 and $556 \mathrm{~nm}$ ). (c) Calibration curves obtained with $0.62 \mathrm{nmol}$ ( $25 \mu \mathrm{L}$ of $25 \mu \mathrm{M}$ solution) of reduced cytochrome $c$ either (blue) directly in PBS buffer solution (no insert) or (red) crosslinked into a glass fiber enhancer insert, and exposed to varying concentrations of $\mathrm{H}_{2} \mathrm{O}_{2}(0.5 \mu \mathrm{M}$ to $1 \mathrm{mM}$ contained in $100 \mu \mathrm{L}$ sample). The corresponding graph clearly shows the gain in sensitivity and limit of detection obtained when enhancer inserts are placed into the wells. Response times were 30 and 5 min for solution bioassay and insertbased biosensor, respectively. Error bars indicate the calculated standard deviation $(n=3)$. (For interpretation of the references to color in this figure legend, the reader is referred to the web version of this article.) et al. as the basis for an absorbance assay in which the decrease of ferrocytochrome $c$ peak at $550 \mathrm{~nm}$ was directly related to the concentration of $\mathrm{H}_{2} \mathrm{O}_{2}$ in the cuvette (Vandewalle and Petersen, 1987). Therefore, in our study, glass fiber inserts were modified with reduced cytochrome $c(0.62 \mathrm{nmol})$ and the decrease in the peak height $(550 \mathrm{~nm})$ subsequent to sample processing was calculated considering the baseline between 542 and $556 \mathrm{~nm}$ (intersection points for reduced and oxidized cytochrome $c$ spectra). Similar absorbance value calculations were performed in control experiment, where the same amount of reduced cytochrome $c(25 \mu \mathrm{L}$ of $25 \mu \mathrm{M}$ solution) was added into PBS-containing standard wells in the absence of any absorbance enhancer insert. The corresponding sigmoidal dose-response curves shown in Fig. 3c were analyzed using the four-parameter log-logistic (4-PL) fitting approach and the calculated parameters are summarized in Table S1 (Findlay and

a

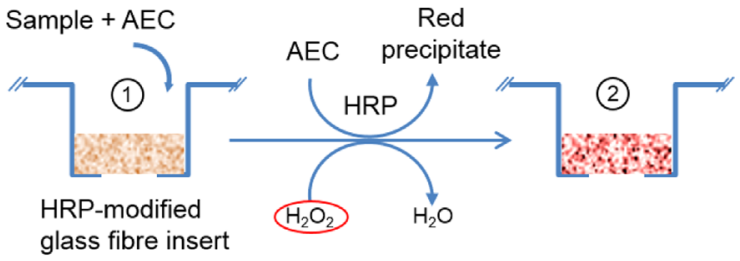

b

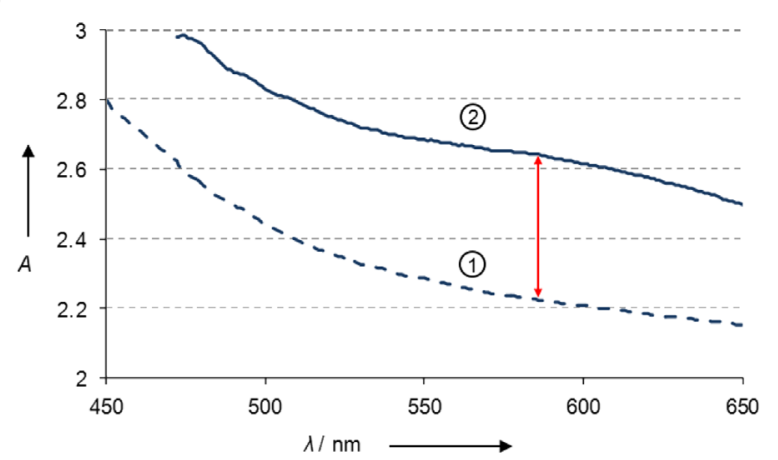

C

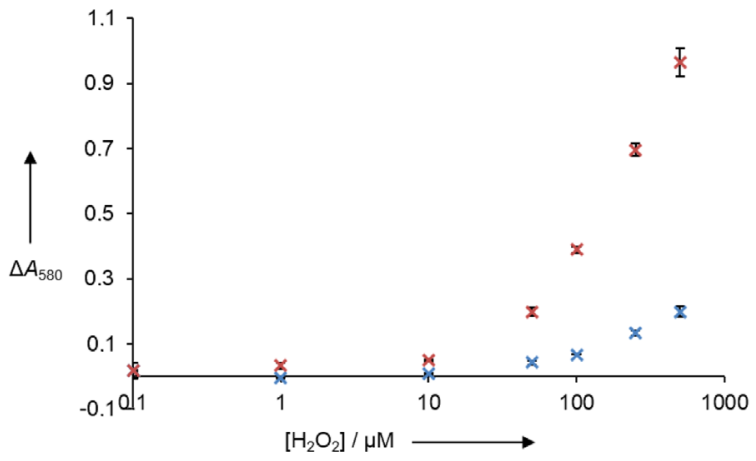

Fig. 4. (a) Biosensor configuration for $\mathrm{H}_{2} \mathrm{O}_{2}$ detection based on the enhanced absorbance measurement of the catalytic conversion of HRP co-substrate, AEC, into an insoluble reddish product. Clean glass fiber inserts are modified with HRP and washed several times prior to experiments. (b) The production of red precipitate in the presence of $\mathrm{H}_{2} \mathrm{O}_{2}$ induces an increase in the measured absorbance $\left(A_{\max }=580 \mathrm{~nm}\right)$. (c) Calibration curves were performed using $0.6 \mathrm{nmol}$ of HRP ( $25 \mu \mathrm{L}$ of $1 \mathrm{mg} \mathrm{mL}^{-1}$ solution) either (blue) directly in acetate buffer solution (no insert) or (red) crosslinked into a glass fiber enhancer insert, and exposed to varying concentrations of $\mathrm{H}_{2} \mathrm{O}_{2}(1-500 \mu \mathrm{M}$ contained in $100 \mu \mathrm{L}$ sample) in the presence of AEC ( $142 \mathrm{nmol}$ ). The graphs indicate a significant improvement of the biosensor analytical performances in the case of HRP-modified enhancer insert. Response times: 15 and $5 \mathrm{~min}$ for solution assay and insert-based biosensor, respectively. Error bars indicate the calculated standard deviation $(n=3)$. (For interpretation of the references to color in this figure legend, the reader is referred to the web version of this article.) 
Dillard, 2007). Evidently, a significant absorbance gain is visible in the case of cytochrome $c$ oxidation that takes place in the enhancer inserts, as reflected by the corresponding signal amplitude value (top-bottom) being more than 6-fold greater than for the membraneless bioassay. The limit-of-detection was calculated (response at 3-folds the standard deviation for blank) for both configurations with and without the enhancer inserts and found to be 3 and $147 \mu \mathrm{M}$, respectively. This significant improvement of the limit-ofdetection was obtained in spite of the increase of the standard deviation values calculated for the membrane insert-based biosensor. In order to evaluate comparatively the sensitivity of the two detection approaches, the sensitivity $S_{50}$ at the inflection point of the curve defined as the ratio of the half-maximum absorbance to the corresponding $\mathrm{H}_{2} \mathrm{O}_{2}$ concentration, $\mathrm{C}_{50}$, was assessed. In comparison to the membrane-less bioassay configuration the calculated $S_{50}$ values show a sensitivity enhancement of more than 40 folds in the case of the membrane insert biosensor. In other words, the analysis of the doseresponse curves provides a clear indication that the developed biosensor approach improves significantly the analytical performances of the detection assay. The overall analytical improvement of the detection assay has to be attributed not only to the optical enhancement due to the elongation of the light path length but also to the efficient mass transport enabled by the vertical flow generated through the $\mathrm{H}_{2} \mathrm{O}_{2}$-sensitive enhancer insert. With this practical procedure the whole sample volume is forced to flow through the membrane and thus no limitation associated to low diffusion rate is observed. Consequently, the response time for the enhanced assay became shorter in comparison to the assay in solution, dropping down from 30 to $5 \mathrm{~min}$. As a general comment, this aspect strongly emphasizes the adaptability of biosensors versus bioassays due to the fact that the recognition biomolecule is retained on the solid phase transducer and therefore larger volumes of sample can be eluded with no dilution issue. Besides, the versatility of the detection enhancement approach was explored in another biosensor configuration that involved the HRP enzymatic activity. HRP is a hemoprotein $(40 \mathrm{kDa})$ frequently used as a label protein in ELISA tests since it catalyzes the oxidation of a series of chromogenic or fluorescent compounds in the presence of $\mathrm{H}_{2} \mathrm{O}_{2}$.

In contrast to the biosensing approach previously described which relies on the detection of spectral changes affecting the immobilized protein, i.e. cytochrome $c$ oxidation, the detection principle now consists of measuring the development of a product enzymatically catalyzed. For the latter strategy it is required that the enzymatic product exhibits a poor solubility in water and forms a colored precipitate which remains inside the glass fiber membrane insert, that is where optical path enhancement occurs. In this context, it appeared appropriate to choose AEC, a commonly used chromogen for staining peroxidases in tissue and cell preparations or even for characterizing catalytic activity of immobilized HRP monolayers (Suárez et al., 2007). As depicted in Fig. 4a and $\mathrm{b}$, for this second biosensor configuration the membrane inserts were biofunctionalized with HRP $(0.62 \mathrm{nmol})$ and the progressive development of reddish precipitate in the presence of $\mathrm{H}_{2} \mathrm{O}_{2}$ and $\mathrm{AEC}$ was measured as an absorbance increase at $580 \mathrm{~nm}$. Similarly as before, control experiments were performed with the same amount of HRP in solution ( $25 \mu \mathrm{L}$ of $\left.1 \mathrm{mg} \mathrm{mL}^{-1}\right)$ in order to evaluate the analytical gain generated in the biosensor approach. The analysis of the corresponding dose-response curves via the 4-PL model equation reveals that the maximum absorbance value obtained in the membrane insert configuration is more than 3 fold higher than for the bioassay in solution. However, the calculated limit of detection was found to be in the same order of magnitude, 1 and $3 \mu \mathrm{M}$ for the bioassay in solution and the membrane insert biosensor, respectively. In terms of sensitivity, the calculated values for $S_{50}$, as earlier defined, indicate an enhancement in sensitivity of 7 folds in the case of the membrane insert approach. Moreover, the benefit in generating an active mass transport through the sensitive insert was manifested in the decrease of response time from 15 to $5 \mathrm{~min}$. Even though the analytical gain arises clearly from this comparative study it is noticeable that a more dramatic contrast was evidenced with the earlier biosensor scheme based on cytochrome $c$ oxidation. One possible explanation is that some AEC product generated in the membrane insert might have been washed away during sample elution. This signal loss is unlikely to occur in the cytochrome $c$-based biosensor since the protein is covalently attached to the solid phase insert.

\section{Conclusion}

In conclusion, the possibility to employ the absorbance enhancement phenomenon as a novel transduction principle suitable for microplate format assays has been successfully demonstrated. Inserts made of glass fiber membrane generate a significant absorbance gain which is dependent on the refractive index difference between the membrane and the surrounding medium. Therefore, the biofunctionalization of membrane inserts with $\mathrm{H}_{2} \mathrm{O}_{2}$-sensitive proteins gave rise to the development of biosensors exhibiting improved analytical performances. Particularly in the case of cytochrome $c$-based biosensor the measured sensitivity was 40 folds greater than for the corresponding bioassay in solution while the limit-of-detection dropped down by a factor of 50 folds. In addition to their optical enhancement properties the membrane inserts act as a solid phase through which the whole sample volume can be easily eluded, resulting in shorter response time, while contributing to the overall improvement of the analytical gain. Finally, this simple biosensor strategy based on both absorbance enhancement and solid-phase configuration enabled by the use of glass fiber inserts appears to be particularly appropriate to improve the analytical performances of existing bioassays via their effective conversion into highly sensitive biosensors.

\section{Acknowledgment}

This work was supported by the Swiss National Science Foundation (Projects 406440_131282 and 406440_131280 in the framework of the Swiss National Research Program NRP 64).

\section{Appendix A. Supplementary material}

Supplementary data associated with this article can be found in the online version at http://dx.doi.org/10.1016/j.bios.2013.12.063.

\section{References}

Chen, C., Song, G.T., Ren, J.S., Qu, X.G., 2008. Chem. Commun. 46, 6149-6151.

Ding, N., Yan, N., Ren, C., Chen, X., 2010. Anal. Chem. 82 (13), 5897-5899.

Findlay, J.W.A., Dillard, R.F., 2007. AAPS J. 9 (2), E260-E267.

Gao, L.Z., Zhuang, J., Nie, L., Zhang, J.B., Zhang, Y., Gu, N., Wang, T.H., Feng, J., Yang, D.L Perrett, S., Yan, X., 2007. Nat. Nanotechnol. 2 (9), 577-583.

Gillespie, K.M., Ainsworth, E.A., 2007. Nat. Protoc. 2 (4), 871-874.

Grumann, M., Steigert, J., Riegger, L., Moser, I., Enderle, B., Riebeseel, K., Urban, G., Zengerle, R., Ducree, J., 2006. Biomed. Microdevices 8 (3), 209-214.

John, S., Pang, G., 1996. Phys. Rev. A 54 (4), 3642-3652.

Kuang, Y., Salem, N., Wang, F.J., Schomisch, S.J., Chandramouli, V., Lee, Z.H., 2007. J. Biochem. Biophys. Methods 70 (4), 649-655.

Liu, J.W., Lu, Y., 2003. J. Am. Chem. Soc. 125 (22), 6642-6643.

Liu, J.W., Mazumdar, D., Lu, Y., 2006. Angew. Chem. Int. Ed. 45 (47), 7955-7959.

Malcik, N., Ferrance, J.P., Landers, J.P., Caglar, P., 2005. Actuator B Chem. 107 (1), 24-31.

Mao, X., Ma, Y.Q., Zhang, A.G., Zhang, L.R., Zeng, L.W., Liu, G.D., 2009. Anal. Chem. 81 (4), 1660-1668.

Martinez, A.W., 2011. Bioanalysis 3 (23), 2589-2592.

Plested, J.S., Coull, P.A., Gidney, M.A.J., 2003. Methods Mol. Med. 71, 243-261. 
Popescu, G., Dogariu, A., 1999. Opt. Lett. 24 (7), 442-444.

Renneberg, R., Pfeiffer, D., Lisdat, F., Wilson, G., Wollenberger, U., Ligler, F., Turner, A.P.F., 2008. In: Renneberg, R., Lisdat, F. (Eds.), Biosensing for the 21st Century. Springer-Verlag, Berlin, pp. 1-18

Sato, K., Hosokawa, K., Maeda, M., 2003. J. Am. Chem. Soc. 125 (27), 8102-8103.

Song, Y., Wei, W., Qu, X., 2011. Adv. Mater. 23 (37), 4215-4236.

Suárez, G., Jackson, R.J., Spoors, J.A., McNeil, C.J., 2007. Anal. Chem. 79 (5), 1961-1969.
Suárez, G., Santschi, C., Dutta-Gupta, S., Juillerat-Jeanneret, L., Martin, O.J.F., 2012. Procedia Eng. 47 (0), 1281-1283.

Suárez, G., Santschi, C., Slaveykova, V.I., Martin, O.J.F., 2013. Sci. Rep. 3, 3447.

Svensson, T., Shen, Z.J., 2010. Appl. Phys. Lett. 96, 2.

Vandewalle, P.L., Petersen, N.O., 1987. FEBS Lett. 210 (2), 195-198.

Zhang, J.Z., 2006. Anal. Sci. 22 (1), 57-60. 